\title{
THE IONIZATION AND THERMAL STRUCTURE OF NGC 2392
}

\section{AND NGC 3242}

\author{
XIAO-WEI LIU and JOHN DANZIGER \\ European Southern Observatory, Garching bei München, FRG
}

In Liu and Danziger(1992b) we present $T_{e}$ derived from the Balmer discontinuities for 14 objects by long slit spectrophotometry. It is found that on the average the $T_{e}$ derived in this way are systematically lower than those derived the forbidden line ratios, indicating the presence of large temperature fluctuations(Peimbert 1967, 1971). The spectra obtained have been analyzed point-by-point along the slit for NGC 2392 and NGC 3242 , for which $t^{2}=0.13$ and 0.058 , respectively, as measured on the summed spectra integrated along the slits.

For each pixel along the slit, we derive the $N_{e}$ from [OII] $I(\lambda 3729) / I(\lambda 3726)$ and [ArIV] $I(\lambda 4711) / I(\lambda 4740)$, the $T_{e}$ from the [OIII] forbidden line ratio and from the observed Balmer discontinuity, $\mathrm{N}\left(\mathrm{He}^{+}\right) / \mathrm{H}^{+}$and $\mathrm{N}\left(\mathrm{He}^{++}\right) / \mathrm{H}^{+}$respectively from HeI $\lambda 4471$ and HeII $\lambda 4686$. In the case of NGC 2392, $T_{e}$ derived the [NII] lines is also obtained. Uniform reddening has been assumed in both NGC 3242 and NGC 2392 for which we have adopted $c=0.12$ and $c=0.22$ respectively.

1. NGC 3242 The [OIII] temperature is quite uniform along the slit. In contrast, the Balmer temperature show large amplitude variations. Within the bright shell, where both the HI $\beta$ and HeII $\lambda 4686$ are strongest, $T_{e}(B a c)$ are close to $T_{e}\left(\mathrm{OIII}_{n a}\right)$, whereas both inside and outside the bright shell $T_{e}(B a c)$ drops rapidly below $T_{e}\left(\mathrm{OIII}_{n a}\right)$ by as much as $3000 \mathrm{~K}$. It seems such large variations of the Balmer temperature is responsible for the large value of $t^{2}=0.058$ found for this object.

2. NGC 2392 It has rather complicated structure both in ionization and thermal structure. The most striking feature is that its ionization degree increases outward. The ionization degree and [OIII], [NII] temperatures are lowest within the central bright disk. In contrast here the Balmer temperatures are highest and comparable to that of [OIII]. The densities in this central disk seems quite uniform, with $N_{e}$ about $2200 \mathrm{~cm}^{-3}$. Outside the central disk, the $N_{e}$ decreases rapidly to values below $600 \mathrm{~cm}^{-3}$, then flattens and decreases to nearly zero, although with some fluctuations. The intensity of HeII $\lambda 4686$ line charges almost exactly oppositely as the $N_{e}$ does. At the meantime, the [OIII] and [NII] temperatures increase rapidly whereas the Balmer temperature decreases. At some positions [OIII] temperature reaches values as high as $18000 \mathrm{~K}$, about $4000 \mathrm{~K}$ higher than those within the central disk. The Balmer temperatures at these positions are only about $10000 \mathrm{~K}$. Throughout the selected slit, the [NII] temperatures are systematically lower than that of [OIII]. Both of them show a similar variation pattern.

\section{References}

Liu, X. W. and Danziger, J., 1992a, submitted.

Liu, X. W. and Danziger, J., 1992b, to be submitted.

Peimbert, M., 1967, Ap.J., 150, 825.

Peimbert, M., 1971, Bol. Observ. de Tonantzintla Y Tacubaya, 6, 29. 